\title{
Discrepancy in pathology reports upon second review of radical orchiectomy specimens for testicular germ cell tumors
}

Gregory J. Nason ${ }^{1}$; Joan Sweet ${ }^{2}$; Lauren Landoni ${ }^{1}$; Ricardo Leao ${ }^{1,3}$; Lynn Anson-Cartwright ${ }^{1}$; Spencer Mok ${ }^{1}$; Vanessa Guzylak ${ }^{1}$; Andrea D’Angelo; Zhi Yi Fang ${ }^{1}$; Ilana Geist ${ }^{1}$; Padraig Warde $^{4}$; Michael A.S. Jewett ${ }^{1}$; Robert J. Hamilton ${ }^{1}$

${ }^{1}$ Division of Urology, Department of Surgery, Princess Margaret Cancer Centre, Toronto, ON, Canada; ${ }^{2}$ Department of Pathology and Lab Medicine, University Health Network, University of Toronto, Toronto, ON, Canada; ${ }^{3}$ Faculty of Medicine; University of Coimbra, Portugal; Clinical Academic Center of Coimbra, Portugal; ${ }^{4}$ Department of Radiation Oncology, Princess Margaret Cancer Centre, Toronto, ON, Canada

Funding: This study was supported by Dell'Elce family fund; the Canadian Urological Association Scholarship Foundation Career Development Award (RJH); and the Astellas Urologic Research and Innovation Fund.

Cite as: Can Urol Assoc J 2020 June 16; Epub ahead of print. http://dx.doi.org/10.5489/cuaj.6481

Published online June 16, 2020

$* * *$

\section{Abstract}

Introduction: We sought to evaluate the discrepancies between primary pathology report and second pathology review of radical orchiectomy (RO) specimens.

Methods: A retrospective review was performed of RO specimens from the Ontario Cancer Registry. All cases required both a primary pathology report and a second pathology review from another institution. Histopathological variables assessed included histological subtype and components of mixed germ cell tumor (GCT), pathological tumor (pT) stage, lymphovascular invasion (LVI), spermatic cord invasion, and surgical margin.

Results: Between 1994 and 2015, 5048 ROs were performed with 2719 (53.9\%) seminoma and $2029(40.2 \%)$ non-seminoma. Of these, 519 (10.3\%) received a second pathology review. There was concordance between primary pathology report and second pathology review in $326(62.8 \%)$ cases. The most common discrepancies involved a change in pT stage $(n=148$, $28.5 \%)$, with upstaging in $83(16 \%)$ and downstaging in $65(12.5 \%)$ cases relative to the original pT stage. The second most common discrepancy regarded the reporting of LVI $(\mathrm{n}=121,23.3 \%)$, with $62(11.9 \%)$ reporting presence of LVI when the primary pathology report did not. Other discrepancies included a change in the histological subtype in $28(5.4 \%)$ cases and spermatic cord margin status in $5(9.6 \%)$ cases.

Conclusions: Only 10\% of orchiectomy specimens underwent a second pathology review, with nearly $40 \%$ of reviews leading to a meaningful change in parameters. Such variation could lead to incorrect tumor staging, estimate of relapse risk, and inappropriate treatment 


\section{CUAJ - Original Research \\ Nason et al \\ Discrepancies between primary and second pathology reports of RO specimens}

decisions. Expert pathology review of RO specimens should be considered, as it has significant implications for decision making.

\section{Introduction}

Testis cancer is the most common solid organ malignancy in men aged 15 to 44 [1]. Most men present with clinical stage one (CS1) disease, with cancer confined to the testis and no evidence of metastatic disease. In such cases, radical orchiectomy (RO) alone is the mainstay of treatment with ongoing clinical and radiological surveillance [2] and 5-year survival is $99 \%$ [1]. Given the excellent overall survival rate, surveillance is the predominant management strategy for CS1 disease with a concerted awareness to minimize the morbidity of any treatment [3]. As surveillance protocol may be individualized based on risk of relapse [4]- an accurate initial pathological diagnosis is of paramount importance.

The Canadian guidelines for the management of testis cancer recommend that all histological specimens be assessed by a pathologist experienced in testis cancer [5]. They also advise the histopathological report should document the following points: localization and size of the tumour, multiplicity, tumour extension (rete testis, tunica albuginea, tunica vaginalis, epididymis, spermatic cord, scrotum), pT category according to the Union for International Cancer Control (UICC) classification, histological type, the presence or absence of tubular intra-epithelial neoplasia, as well as the presence or absence of vascular invasion of blood or lymphatic vessels. The American Urological Association guidelines state 'an expert review of pathologic specimens should be considered in clinical scenarios where treatment decisions will be impacted' [6]- it could be argued that this is true for all cases. Although the European Association of Urology (EAU) guidelines do not specify a dedicated testis cancer pathologist they suggest mandatory pathological requirements from the histopathological report [7].

Standardized techniques have been established on the handling and processing of RO specimens by both urologists and pathologists [8]. Despite this, numerous studies have highlighted the discrepancies in reporting after a central pathology review [9-12].

There have been many calls for regionalization of testis cancer due to the rarity of the disease and the nuances within its management. The first step in the diagnosis of testis cancer is predominantly a RO- Nayan et al, demonstrated that the institution where the RO is performed did not affect oncological outcomes [13]. Given the importance of the pathological diagnosis- the initiation of regionalization could be a central pathological review.

The aim of this study was to evaluate the discrepancies between primary pathology report and second pathology review of RO specimens in Ontario. 
Discrepancies between primary and second pathology reports of RO specimens

\section{Methods}

A retrospective review was performed of RO specimens performed from the Ontario Cancer Registry using the diagnostic codes 'malignant neoplasm of testis' and 'malignant neoplasm of undescended testis'. Cancer Care Ontario collected all pathology reports. All cases required both a primary pathology report from the institution where the RO was performed and a second pathology review from another institution. Cases were excluded if both reports were not available. Histopathological specimens were not reviewed.

Variations in reporting were recorded. Histopathological variables assessed for comparison included histological subtype and components of mixed germ cell tumor (GCT), pathologic tumor (pT) stage, lymphovascular invasion (LVI), spermatic cord invasion (involved or uninvolved), surgical resection margin (positive or negative).

Categorical variables were summarized with counts and percentages. Statistical analysis was performed using $\chi^{2}$ test and Fisher exact test. Results were considered significant if $\mathrm{p}<0.05$. Statistical analyses were performed using version 9.4 of the SAS system for Windows (2002-2012 SAS Institute, Inc., Cary, NC). The study was approved by the institutional review board (CAPCR \# 14-7509).

\section{Results}

Between 1994 and 2015, 5048 RO were performed in Ontario, 2719 (53.9\%) seminoma and 2029 (40.2\%) nonseminoma. Of these $519(10.3 \%)$ received a second pathology review. The vast majority $(411,79.2 \%)$ of second pathology reviews occurred at two institutions.

However, second pathology reviews occurred at 21 different institutions with a median of 2 (Range1-319) reviews per institution.

There was concordance between the primary pathology report and second pathology review in $326(62.8 \%)$ cases. The most common discrepancy involved a change in pT stage $(\mathrm{n}=148,28.5 \%)$ with the second pathology review leading to upstaging in $83(16 \%)$ cases and down-staging in $65(12.5 \%)$ cases. The details in pT stage change are listed in Table 1.

The second most common discrepancy regarded the reporting of LVI ( $n=121,23.3 \%)$. LVI was reported as present in the primary pathology report and absent in the second pathology review in $58(11.2 \%)$ cases. Conversely, LVI was reported as absent in the primary pathology report and present in the second pathology review in $37(7.1 \%)$ cases. LVI was not commented on in $26(5 \%)$ primary pathology reports but was reported as present in $25(4.8 \%)$ and absent in $1(0.2 \%)$ upon second pathology review.

There was a change in the histological subtype in 28 (5.4\%) cases. The changes were between types of GCT in 23 (4.4\%) cases: seminoma to nonseminoma (NSGCT) in 13 $(2.5 \%)$ cases, NSGCT to seminoma in $8(1.5 \%)$ cases, leydig cell tumor to a seminoma in 1 $(0.2 \%)$ case and NSGCT to a leydig cell tumor in $1(0.2 \%)$ case. There was a change from a GCT (seminoma) to lymphoma in $3(0.6 \%)$ cases. Two $(0.4 \%)$ cases were described as no tumour seen in the primary pathology report but on second pathology review revealed a seminoma in $1(0.2 \%)$ and a NSGCT in the other $(0.2 \%)$, Table 2 . Within mixed GCT, there 
was a change in the components in $146(28.1 \%)$ cases. Embryonal carcinoma was reported present and changed to absent in $13(2.5 \%)$ cases while it was reported absent and changed to present in $31(6 \%)$ cases. Choriocarcinoma was reported present and changed to absent in 19 (3.7\%) cases while it was reported absent and changed to present in $14(2.7 \%)$ cases. Yolk sac tumor was reported present and changed to absent in $10(1.9 \%)$ cases while it was reported absent and changed to present in $60(11.6 \%)$ cases.

There was a change in spermatic cord involvement in $20(3.9 \%)$ cases. The spermatic cord was initially reported as involved and changed to uninvolved in $8(1.5 \%)$ cases while it was reported as uninvolved and changed to involved in $12(2.3 \%)$ cases. Spermatic cord margin status was changed in $5(1 \%)$ cases. The surgical margin was initially reported negative and changed to positive on second pathology review in $2(0.4 \%)$ cases. Conversely, the surgical margin was initially reported positive and changed to negative on second pathology review in $3(0.6 \%)$ cases.

We compared the discordance rates of the primary pathology report and second pathology review based on the two most common second review institutions collectively $(\mathrm{n}=$ $411,79.2 \%)$ and the other 19 second review institutions collectively ( $\mathrm{n}=108,20.8 \%)$, Table 3 . Overall, the discordance rate was significantly lower among pathology reviewed at the two highest-volume centres $(33.1 \%$ vs $63.9 \%, \mathrm{p}=0.0001)$. Driving this difference was discordance in histological subtypes $(3.9 \%$ vs $11.1 \%, \mathrm{p}=0.007)$ and surgical margin involvement $(3.4 \% \mathrm{vs}$ $8.3 \%, \mathrm{p}=0.036$ ) between the two most common second review institutions and the others. There was no difference noted in discordance rates of reporting of LVI $(23.6 \%$ vs $29.5 \%$, $\mathrm{p}=0.315)$ and pathological stage $(26.8 \%$ vs $35.2 \%, \mathrm{p}=0.09)$.

\section{Discussion}

Despite guidelines encouraging second pathology review [5-7]- in Ontario, this only occurred in $10 \%$ of cases. Our study highlights that variations in pathology reporting of RO specimens is not uncommon. We observed discordance between primary pathology reports and second pathology review in almost $40 \%$ of cases and these discrepancies pertained to clinically meaningful parameters including pathological stage in $28 \%$, LVI in $23 \%$, surgical margin status in $9.6 \%$ and histological subtype in 5.4\%. Such discrepancies could lead to inappropriate treatment decisions. There were significantly different discordance rates between the two most common second review institutions, which performed almost $80 \%$ of the reviews and the other 19 institution, with almost a two fold increase in the number of discordant reviews at the less established review institutions.

Central pathology review has clinical consequences and alters management in many cancers. Recently, de Boer et al., reported that central pathology review as part of a high risk endometrial cancer trial resulted in changes to histological type and grade in $43 \%$ of women, an almost identical discrepancy rate to our study, leading to ineligibility for the PORTEC-3 trial in 8\% [14]. Saga et al., demonstrated a 41 discordant rate in prostate biopsy reports for the classification of intermediate risk disease which has significant management implications 
given low risk disease is usually managed with active surveillance whereas radical treatment are offered for intermediate risk disease [15]. Rare tumors such as pediatric bone and soft tissue tumors are often challenging cases for general pathologists. A review of over 1600 such cases reported a diagnosis was suggested in less than half (42\%) and of these there were discrepancies in 29\% [16]. The main reasons for referral for a second pathology review included a self-perceived lack of experience with pediatric mesenchymal tumors, a second opinion requested by the clinician or patient, and a perceived or real need for ancillary studies not available at the referring institution. Given the remarkably different discordant rates at second pathology review between the two most common second review institutions and the others in our series, this highlights the need for a formal pathway of second review directed towards high volume centres.

In testis cancer, certain factors have been identified as predictors of relapse and hence the reporting of these factors is important. There is debate that in the presence of these highrisk factors then patients should be offered adjuvant treatment in a risk-stratified approached. Tumor size was deemed to be the most valuable prognostic factor in a systematic review of CS1 seminoma relapse; rete testis invasion plays a more minor role [17]. For NSGCT- LVI and $>50 \%$ embryonal component have been shown to be predictors of relapse. The risk of relapse with evidence of LVI approaches 30-50\% [18]. The presence of these risk factors has significant implications for the management of CS1 testis cancer. Although surveillance has been largely adopted for CS1 disease, the guidelines still stratify CS1 NSGCT into stage 1A and stage 1B (based upon the presence or absence of LVI). For stage 1A NSGCTsurveillance is uniformly the preferred option however for stage 1B, the AUA, EAU and NCCN guidelines discuss a risk adapted approach as an option on a shared decision basis $[6,7,19]$. Avulova et al., performed a decision analysis of high risk (LVI) NSGCT and found surveillance to be superior to chemotherapy or primary retroperitoneal lymph node dissection when integrating all of possible chance occurrences and survival related morbidities [20]. Although non-risk adapted surveillance is the mainstay for CS1 disease in Canada, adjuvant treatment with chemotherapy is being used routinely in some jurisdictions for CS1 disease with high risk features [21,22]. The high-risk feature of LVI was altered in $23 \%$ following second review in our study.

A major error in pathological interpretation is histological subtype (ie. Seminoma vs. NSGCT) which we observed in $4.4 \%$ of cases. Surveillance schedules are adjusted based upon GCT subtype, with seminomas surveilled with less intensity but followed for longer ( 9 years vs. 5 years) than NSGCT. Thus patients with inaccurate histological subtyping may be surveilled with inappropriate intensity. Worse yet, treatment decisions at relapse are based on understanding of primary pathology. For example, a presumed seminoma relapse is treated with retroperitoneal radiation most commonly at Princess Margaret. This would be largely ineffectual and non-guideline concordant treatment if, in fact, the primary had been an NSGCT. 
Discrepancies between primary and second pathology reports of RO specimens

The status of the spermatic cord margin and spermatic cord invasion was altered in $1 \%$ and $4 \%$ in our cohort- invasion of the spermatic cord is associated with more advanced disease and higher rates of relapse [23] with some institutions advocating hemiscrotectomy or adjuvant radiation.

Previous studies have highlighted the disparities in reporting of primary testis cancer [9-12]. Harari et al, compared the final diagnosis between outside institutions and a central review by expert genitourinary pathologists at Indiana University- a centre well known for testis cancer [11]. Of 221 cases reviewed, 31\% demonstrated some discrepancy with histological subtype (all NSGCT), 22\% in LVI reporting and $9 \%$ regarding spermatic cord invasion. The pathologic stage was altered in $10.4 \%$ with $40 \%$ of these being upstaged. Sharma et al., noted disparities in $21 \%$ of RO specimens resulting in a clinically significant change in $6.8 \%$, defined as change in subtype from seminoma to NSGCT (or vice-versa) or a change in LVI status [10]. The most common discrepancy appears to be differences in reporting LVI. Similar to our findings, Sesterhenn et al, found a discordance rate of $36 \%$ for LVI which correlated with relapse rates as a high risk feature [24].

The current Canadian and American guidelines for testis cancer both make reference to a pathology review by a dedicated or expert uro-pathologist given the implications for further treatment $[5,6]$. While we advocate pathology review at high-volume centres for all orchiectomy specimens, it is important to note that practicing non-risk-adapted active surveillance for CS1 seminoma and NSGCT renders the primary pathology report accuracy less important. We have practiced this way at Princess Margaret since 1980 and this has been echoed by others [3,25]. Non-risk-adapted surveillance is controversial [26], and while endorsed by Canadian guidelines, the American and European guidelines acknowledge that adjuvant treatment may be chosen for higher-risk CSI seminoma and NSGCT. One advantage to a non-risk-adapted approach is that the presence of LVI, the amount of embryonal carcinoma, and the presence of rete testis invasion is inconsequential to the decision to apply adjuvant therapy.

Interobserver variability is well recognized within many facets of diagnostic reporting both in pathology and radiology. Standardized specimen handling, processing and reporting techniques have been developed to try to minimize variability $[8,27]$. Improvements in immunohistochemical staining techniques have increased the diagnostic accuracyparticularly between histological subtypes [27]. Future studies will focus on the use of microRNA as a biomarker which will help in the diagnosis of testis cancer, differentiating subtypes and also in predicting relapse [28].

Recommending RO pathology review is part of a larger discussion of regionalization of care for testis cancer, a prevalent topic at the recent Canadian Testis Cancer Workshop. Cancer Care Ontario (CCO) understands the importance of regionalizing care of rare cancers and has been successful in regionalizing certain cancers to date. The management of sarcoma is one such example [29]. Quality sarcoma care mandates pathology review at the Host Centre by pathologists with a specialty or special interest in sarcoma working at centres with 
access to molecular diagnostics, site specific imaging, advanced limb salvage and abdominal surgery, high precision radiotherapy, and the delivery of complex chemotherapy regimens. Most aspects of subsequent care are also mandated to occur at designated host sites.

The National Health Service (NHS) in the UK is a similar publicly funded single payer health service to Canada. In 2002, the National Institution for Clinical Excellence (NICE) released a manual 'Improving outcomes in urological cancers' [30]. Similar to regionalization- they recommended the development of networks to provide and co-ordinate a wide range of services for patients with urological cancers within a defined geographical area. The NICE guideline stipulates the initial diagnosis and management (radical orchiectomy) can be provided locally and then referral to the testis cancer network within 24 hours after surgery unless there is evidence of de novo metastatic disease.

This retrospective review of a population database has a number of limitations. Firstly, these databases may not capture all second review cases however it is likely that any further cases would mirror these findings. Secondly, given that $\sim 10 \%$ of cases received a second review- our main concern is that the other $90 \%$ of cases may have some degree of error within their primary pathology report which may have impacted treatment decisions. Thirdly, the Canadian guidelines recommend a review of all specimens by a pathologist experienced in testis cancer- although the majority of second reviews occurred at two high volume centres with dedicated genitourinary pathologist, we do not have details of the experience level of the primary or secondary reporting pathologist in many of these institutions. Finally, this study was based upon a review of pathology reporting- none of the specimens were reviewed as a quality assessment.

\section{Conclusions}

Despite international guidelines encouraging pathology review in testicular cancer, in Ontario, only $10 \%$ of specimens are reviewed. We observed that in up to $40 \%$ of cases discrepancies are noted in meaningful pathological parameters and such discrepancies could lead to inaccurate categorization of subtype (e.g. seminoma vs. NSGCT), staging, relapse risk estimate and inappropriate treatment decisions. Given the relative rarity of testicular germ cell tumors, pathology review of orchiectomy specimens at an experienced centre should be considered because of the significant implications for decision making. 
Discrepancies between primary and second pathology reports of RO specimens

\section{References}

1. http://www.cancer.ca/en/cancer-information/cancer-type/testicular/statistics (Accessed 27th May 2019)

2. Goldberg H, Madhur N, Hamilton RJ. Conditional risk of relapse in patients with germ cell testicular tumors: personalizing surveillance in clinical stage 1 disease. Curr Opin Urol. 2018 Sep;28(5):454-460

3. Sturgeon JF, Moore MJ, Kakiashvili DM, et al. Non-risk-adapted surveillance in clinical stage I nonseminomatous germ cell tumors: the Princess Margaret Hospital's experience. Eur Urol. 2011 Apr;59(4):556-62.

4. Nayan M, Jewett MA, Hosni A, et al. Conditional Risk of Relapse in Surveillance for Clinical Stage I Testicular Cancer. Eur Urol. 2017 Jan;71(1):120-127.

5. Wood L, Kollmannsberger C, Jewett M, et al. Canadian consensus guidelines for the management of testicular germ cell cancer. Can Urol Assoc J. 2010 Apr;4(2):e19-38.

6. Stephenson A, Eggener SE, Bass EB, et al. Diagnosis and Treatment of Early Stage Testicular Cancer: AUA Guideline. J Urol. 2019 May 6:101097JU0000000000000318.

7. Albers P, Albrecht W, Algaba F, et al. Guidelines on Testicular Cancer: 2015 Update. Eur Urol. 2015 Dec;68(6):1054-68.

8. Winstanley AM, Mikuz G, Debruyne F, et al. Handling and reporting of biopsy and surgical specimens of testicular cancer. Eur Urol. 2004 May;45(5):564-73. Review.

9. Delaney RJ, Sayers CD, Walker MA, et al. The continued value of central histopathological review of testicular tumours. Histopathology. 2005 Aug;47(2):1669.

10. Sharma P, Dhillon J, Agarwal G, et al. Disparities in Interpretation of Primary Testicular Germ Cell Tumor Pathology. Am J Clin Pathol. 2015 Aug;144(2):289-94.

11. Harari SE, Sassoon DJ, Priemer DS, et al. Testicular cancer: The usage of central review for pathology diagnosis of orchiectomy specimens. Urol Oncol. 2017 Oct;35(10): 605.e9-605.e16.

12. Lee AH, Mead GM, Theaker JM. The value of central histopathological review of testicular tumours before treatment. BJU Int. 1999 Jul;84(1):75-8.

13. Nayan M, Jewett MA, Anson-Cartwright L, et al. The association between institution at orchiectomy and outcomes on active surveillance for clinical stage I germ cell tumours. Can Urol Assoc J. 2016 May-Jun;10(5-6):204-209.

14. de Boer SM, Wortman BG, Bosse T, et al. Clinical consequences of upfront pathology review in the randomised PORTEC-3 trial for high-risk endometrial cancer. Ann Oncol. 2018 Feb 1;29(2):424-430.

15. Soga N, Yatabe Y, Kageyama T, et al. Review of Bioptic Gleason Scores by Central Pathologist Modifies the Risk Classification in Prostate Cancer. Urol Int. 2015;95(4):452-6.

16. Al-Ibraheemi A, Folpe AL. Voluntary Second Opinions in Pediatric Bone and Soft Tissue Pathology: A Retrospective Reviewof 1601 Cases From a Single Mesenchymal Tumor Consultation Service. Int J Surg Pathol. 2016 Dec;24(8):685691.

17. Zengerling F, Kunath F, Jensen K, et al. Prognostic factors for tumor recurrence in 
patients with clinical stage I seminoma undergoing surveillance-A systematic review. Urol Oncol. 2018 Oct;36(10):448-458.

18. Tandstad T, Dahl O, Cohn-Cedermark G, et al. Risk-adapted treatment in clinical stage I nonseminomatous germ cell testicular cancer: the SWENOTECA management program. J Clin Oncol. 2009 May 1;27(13):2122-8.

19. Gilligan T, Lin DW, Aggarwal R, et al. Testicular Cancer, Version 1.2019, National Comprehensive Care Network. 2019.

20. Avulova S, Allen C, Morgans A, et al. Decision analysis defining optimal management of clinical stage 1 high-risk nonseminomatous germ cell testicular cancer with lymphovascular invasion. Urol Oncol. 2018 Jul;36(7):342.e1-342.e6.

21. Huddart RA, Reid AM. Adjuvant Therapy for Stage IB Germ Cell Tumors: One versus Two Cycles of BEP. Adv Urol. 2018 Apr 2;2018:8781698.

22. Vidal AD, Thalmann GN, Karamitopoulou-Diamantis E, et al. Long-term outcome of patients with clinical stage I high-risk nonseminomatous germ-cell tumors 15 years after one adjuvant cycle of bleomycin, etoposide, and cisplatin chemotherapy. Ann Oncol. 2015 Feb;26(2):374-7

23. Sanfrancesco JM, Trevino KE, Xu H, et al. The Significance of Spermatic Cord Involvement by Testicular Germ Cell Tumors: Should We Be Staging Discontinuous Invasion From Involved Lymphovascular Spaces Differently From Direct Extension? Am J Surg Pathol. 2018 Mar;42(3):306-311.

24. Sesterhenn IA, Weiss RB, Mostofi FK, et al. Prognosis and other clinical correlates of pathologic review in stage I and II testicular carcinoma: a report from the Testicular Cancer Intergroup Study. J Clin Oncol. 1992 Jan;10(1):69-78.

25. Hamilton RJ, Nayan M, Anson-Cartwright L, et al. Treatment of Relapse of Clinical Stage I Nonseminomatous Germ Cell Tumors on Surveillance. J Clin Oncol. 2019 Aug 1;37(22):1919-1926.

26. Nason GJ, Leao R, Hamilton RJ. Active surveillance in stage 1 disease: standard of care independent of risk factors? Curr Opin Urol. 2019 Dec 12. doi: 10.1097/MOU.0000000000000708. [Epub ahead of print]

27. Verrill C, Perry-Keene J, Srigley JR, et al. Intraoperative Consultation and Macroscopic Handling: The International Society of Urological Pathology (ISUP) Testicular Cancer Consultation Conference Recommendations. Am J Surg Pathol. 2018 Jun;42(6):e33-e43.

28. Leão R, Ahmad AE, Hamilton RJ. Testicular Cancer Biomarkers: A Role for Precision Medicine in Testicular Cancer. Clin Genitourin Cancer. 2019 Feb;17(1):e176-e183.

29. Provincial Sarcoma Services Plan. https://www.cancercareontario.ca/sites/ccocancercare/files/assets/CCOSarcomaServic esPlan.pdf

30. https://www.nice.org.uk/guidance/csg2/resources/improving-outcomes-in-urologicalcancers-pdf-773372413 
Figures and Tables

\begin{tabular}{|c|c|c|}
\hline Primary review & Secondary review & n (\%) \\
\hline \multicolumn{2}{|l|}{ Upstage } & $83(16)$ \\
\hline pTx & pT1 & $23(4.4)$ \\
\hline $\mathrm{pTx}$ & pT2 & $2(0.4)$ \\
\hline pTx & pT3 & $1(0.2)$ \\
\hline pT1 & pT2 & $49(9.4)$ \\
\hline pT1 & pT3 & $1(0.2)$ \\
\hline pT2 & pT3 & $7(1.3)$ \\
\hline \multicolumn{2}{|l|}{ Downstage } & $65(12.5)$ \\
\hline pT1 & pTx & $1(0.2)$ \\
\hline pT2 & pTx & $2(0.4)$ \\
\hline pT2 & $\mathrm{pT} 1$ & $53(10.2)$ \\
\hline pT3 & $\mathrm{pT} 1$ & $1(0.2)$ \\
\hline pT3 & pT2 & $7(1.3)$ \\
\hline pT4 & pT3 & $1(0.2)$ \\
\hline
\end{tabular}

\begin{tabular}{|c|c|c|}
\hline Primary review & Second review & n (\%) \\
\hline GCT to GCT & 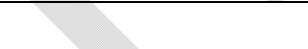 & \\
\hline Seminoma & NSGCT & $13(2.5)$ \\
\hline NSGCT & Seminoma & $8(1.5)$ \\
\hline Leydig cell tumor & Seminoma & $1(0.2)$ \\
\hline NSGCT & Leydig cell tumor & $1(0.2)$ \\
\hline \multicolumn{3}{|l|}{$\begin{array}{l}\text { GCT to other } \\
\text { malignancy }\end{array}$} \\
\hline Seminoma & Lymphoma & $3(0.6)$ \\
\hline \multicolumn{3}{|l|}{ No tumor to GCT } \\
\hline No tumor & Seminoma & $1(0.2)$ \\
\hline No tumor & NSGCT & $1(0.2)$ \\
\hline
\end{tabular}

GCT: germ cell tumor; NSGCT: non-seminomatous germ cell tumor. 
Discrepancies between primary and second pathology reports of RO specimens

\begin{tabular}{|c|c|c|c|}
\hline & $\begin{array}{l}\text { Two most common institutions } \\
\qquad n=411\end{array}$ & $\begin{array}{l}\text { Other } 19 \text { institutions } \\
\qquad n=108\end{array}$ & $\mathbf{p}$ \\
\hline Overall discordant rate & $136(33.1 \%)$ & $69(63.9 \%)$ & 0.0001 \\
\hline Histological subtype & $16(3.9 \%)$ & $12(11.1 \%)$ & 0.007 \\
\hline LVI involvement & $97(23.6 \%)$ & $31(29.5 \%)$ & 0.315 \\
\hline Pathologic pT stage & $110(26.8 \%)$ & $38(35.2 \%)$ & 0.09 \\
\hline $\begin{array}{l}\text { Surgical margin } \\
\text { involvement }\end{array}$ & $14(3.4 \%)$ & $9(8.3 \%)$ & 0.036 \\
\hline
\end{tabular}

LVI: lymphovascular invasion. 